\title{
Microplásticos en peces marinos de importancia económica en Lima, Perú
}

\author{
Microplastics in marine fishes of economic importance in Lima, Peru \\ José Iannacone ${ }^{1,2,3,7}$, Fabiola Principe ${ }^{3}$, David Minaya ${ }^{3}$, Grober Panduro, ${ }^{4,5}$, \\ Mario Carhuapoma ${ }^{6}$, Lorena Alvariño ${ }^{3}$
}

\section{Resumen}

\begin{abstract}
Se evaluó los microplásticos (MP) presentes en cinco peces de importancia económica en el Perú: Sciaena deliciosa (Tschudi, 1846) (Sciaenidae) «lorna», Isacia conceptionis (Cuvier, 1830) (Haemulidae) «cabinza», Scartichthys gigas (Steindachner, 1876) (Blenniidae) «borrachito», Mugil cephalus (Linnaeus, 1758) (Mugilidae) «lisa»y Scomber japonicus (Houttuyn, 1782) (Scombridae) «caballa». El contenido estomacal y branquial de cada pez fue sometido a digestión con $\mathrm{KOH}$ al $10 \%$, seguido por un proceso de filtración para la búsqueda de MP. Los MP fueron clasificados por color y por forma. La contaminación por MP encontrados en el tracto digestivo de $S$. gigas presentaron valores mayores que los encontrados en las otras cuatro especies. No se observaron diferencias en la cantidad de MP registrados en las branquias entre especies. Solo se observó una correlacion positiva entre la longitud corporal de I. conceptionis y MP del tracto digestivo/pez, y entre el peso de M. cephalus y MP del tracto digestivo/pez. El azul, negro y blanco fueron los colores dominantes del los MP a nivel del tracto digestivo, y el azul, negro y fucsia en las branquias. A nivel del tracto digestivo, los tipos de MP fueron fibra, fragmento y film y en branquias solo se hallaron fibras.
\end{abstract}

Palabras clave: ambiente marino, contaminación, microplásticos, perciformes, tracto digestivo

${ }^{1}$ Laboratorio de Parasitología, Facultad de Ciencias Biológicas, Universidad Ricardo Palma, Lima, Perú

${ }^{2}$ Laboratorio de Ingeniería Ambiental, Facultad de Ciencias Ambientales, Coastal Ecosystems of Peru Research Group (COEPERU), Universidad Cientifica del Sur, Lima, Perú

${ }^{3}$ Laboratorio de Ecología y Biodiversidad Animal (LEBA), Facultad de Ciencias Naturales y Matemática, Grupo de Investigación en Sostenibilidad Ambiental (GISA), Escuela Universitaria de Posgrado, Universidad Nacional Federico Villarreal, Lima, Perú

${ }^{4}$ Negocios Amazónicos Sustentables EIRL, Pucallpa, Perú

${ }^{5}$ Universidad Nacional de Ucayali, Pucallpa, Perú

${ }^{6}$ Facultad de Farmacia y Bioquímica. Universidad Nacional Mayor de San Marcos, Lima, Perú

${ }^{7}$ E-mail: joseiannacone@gmail.com

Recibido: 3 de agosto de 2020

Aceptado para publicación: 9 de enero de 2021

Publicado: 24 de abril de 2021 
The microplastics (PM) present in five fish species of economic importance in Peru were evaluated: Sciaena deliciosa (Tschudi, 1846) (Sciaenidae) «Lorna drum», Isacia conceptionis (Cuvier, 1830) (Haemulidae) «Cabinza grunt», Scartichthys gigas (Steindachner, 1876) (Blenniidae) «Giant blenny», Mugil cephalus (Linnaeus, 1758) (Mugilidae) «flathead grey mullet» and Scomber japonicus (Houttuyn, 1782) (Scombridae) «Chub mackerel». The stomach and gill contents of each fish were digested with $10 \%$ $\mathrm{KOH}$, followed by a filtration process to search for PM. The MPs were classified by color and by shape. The contamination by PM found in the digestive tract of S. gigas presented higher values than those found in the other four species. No differences were observed in the amount of MP recorded in the gills between species. A positive correlation was only observed between the body length of I. conceptionis and MP of the digestive tract/ fish, and between the weight of M. cephalus and MP of the digestive tract/fish. Blue, black and white were the dominant colours of MP in the digestive tract, and blue, black and fuchsia in gills. At the level of the digestive tract, the types of PM were fibre, fragments and film and in gills only fibres were found.

Key words: digestive tract, marine environment, microplastics, perciform, pollution

\section{INTRODUCCIÓN}

Los microplásticos (MP) son partículas sólidas sintéticas o de matriz polimérica, de forma regular o irregular, menores a $5 \mathrm{~mm}$ e insolubles en agua (Frias y Nash, 2019). Estas partículas resultan de la liberación directa de plásticos, de pellets de diferentes tamaños utilizados para diversos fines en productos de cuidado personal, agentes de limpieza, cosméticos, etc. (Barboza et al., 2019; Bucol et al., 2020). Los MP pueden ser productos de un proceso de fragmentación lenta generado por energías mecánicas y radiación solar que ocurren en el ambiente (Auta et al., 2017; Pozo et al., 2019). Durante la degradación del MP, se pueden liberar sustancias químicas que generan un impacto negativo en los organismos y ecosistemas al cual están expuestos (Royer et al., 2018; Bucol et al., 2020).

La presencia de MP en diferentes ecosistemas del planeta son de preocupación mundial debido a la contaminación que gene- ran al medio ambiente, especialmente al ambiente marino (GESAMP, 2016), dado que se encuentran desde las aguas superficiales hasta el lecho marino y desde zonas costeras hasta los polos (Bergmann et al., 2017; Cincinelli et al., 2017; Maes et al., 2017; Mu et al., 2019; Savoca et al., 2019). Dichas partículas por su pequeño tamaño y densidad relativamente baja contribuyen a su transporte (Cózar et al., 2017; Barboza et al., 2019) y distribución mundial (Cózar et al., 2014; Suaria et al., 2016; Auta et al., 2017), pudiendo permanecer durante muchos años en el ambiente (Strungaru et al., 2018; Barboza et al., 2019) posibilitando la contaminación de una amplia variedad de organismos acuáticos, incluidas especies utilizadas comúnmente en la dieta humana (Gallo et al., 2018; Barboza et al., 2018).

El conocimiento de los posibles efectos de los MP sobre la biota marina se ha incrementado durante la ultima década (Cole et al., 2011; Barboza et al., 2019). La presencia de estas partículas de MP puede afectar a los seres vivos de diversas formas, prin- 
cipalmente: a) al ser ingeridos, b) transferirse a lo largo de la cadena trófica e c) interaccionar en la incorporación de otros contaminantes (Codina-García et al., 2013). La ingestión de desechos plásticos puede causar lesiones internas y bloqueo del tracto gastrointestinal (Lönnstedt y Eklöv, 2016), además de interferir con la mecánica de alimentación (Wang et al., 2019, 2020a). Los riesgos toxicológicos a través de la transferencia de la cadena alimentaria y la bioacumulación puede representar un peligro para los seres humanos (Bucol et al., 2020).

Un amplio espectro de organismos marinos, tales como los corales, moluscos, peces, aves, tortugas e incluso cetáceos, pueden ingerir MP, o bien incorporarlos mediante la ingesta de presas (Anderson et al., 2016; Bucol et al., 2020). McGoran et al. (2017) mencionan que la cantidad de MP encontrados en el tracto digestivo de peces de ambientes acuáticos es elevada; sin embargo, Davison y Asch (2011) encontraron en una incidencia de $9.2 \%$ de MP en el tracto digestivo de 114 peces mesopelagicos; por lo que se deduce que la ingestión de MP por peces se encuentra influenciada por diversos factores, incluido el nivel de contaminación de MP local, la estrategia de alimentación de cada especie de pez y otros factores ambientales (Romeo et al., 2015; Battaglia et al., 2016; Bucol et al., 2020; Wang et al., 2020a,b).

En el Perú se tiene pocos estudios de MP relacionado al ambiente marino. Por ejemplo, en bivalvos (De-la-Torre et al., 2019b, Valencia-Velasco et al., 2020), arena de playas marinas (Purca y Henostroza, 2017; Iannacone et al., 2019; De-la-Torre et al., 2020) y en peces (Ory et al., 2018; De-laTorre, 2019a).

El objetivo de este estudio fue evaluar los MP en peces marinos, Sciaena deliciosa (Tschudi, 1846) (Sciaenidae) «lorna», Isacia conceptionis (Cuvier, 1830) (Haemulidae) «cabinza», Scartichthys gigas (Steindachner, 1876) (Blenniidae) «borrachi- to», Mugil cephalus (Linnaeus, 1758) (Mugilidae) «lisa» y Scomber japonicus (Houttuyn, 1782) (Scombridae) «caballa» de importancia económica en Lima, Perú.

\section{Materiales Y MéTodos}

\section{Área de Estudio y Peces}

Los peces fueron adquiridos semanalmente entre enero y febrero de 2019 de tres vendedores por fecha del terminal pesquero de Villa María del Triunfo (Lima), Perú. Se consiguieron 100 ejemplares de peces marinos de importancia económica, distribuidos en 20 individuos por especie: $S$. deliciosa «lorna», I. conceptionis «cabinza», S. gigas «borrachito», M. cephalus «lisa» y $S$. japonicus «caballa». Los especímenes fueron transportados al laboratorio en contenedores con geles de refrigeración $\left(-20^{\circ} \mathrm{C}\right)$ para su evaluación. Los peces fueron calificados según su hábitat, rol trófico y profundidad en armonía con la información disponible (Stergiou y Karpouzi, 2002; Hayden et al., 2019; Froese y Pauly, 2020).

\section{Extracción de Órganos}

Se obtuvieron medidas morfométricas de cada uno de los peces, tales como talla en base a la longitud total LT $(\mathrm{cm})$ y el peso $(\mathrm{g})$. Se extrajo el tracto digestivo y las branquias. El tracto digestivo se cortó longitudinalmente en secciones de $2 \mathrm{~cm}$ y se vertió en viales rotulados para su almacenamiento en refrigeración a $-20{ }^{\circ} \mathrm{C}$ y posterior procesamiento para la obtención de MP (Bucol et al., 2020).

\section{Microplásticos}

Se siguió el protocolo establecido por Lusher y Hernandez-Milian (2018) con modificaciones, en el proceso de filtración. El área de trabajo fue limpiada con etanol al 70\% filtrado con un papel filtro de $2.5 \mu \mathrm{m}$ de porosidad (Whatman grado 42). Se utilizaron guantes de nitrilo y se emplearon materiales de 
vidrio o metal para evitar la contaminación de MP. El agua utilizada para el lavado de las muestras y preparación de reactivos fue filtrada con papel filtro de $2.5 \mu \mathrm{m}$ de porosidad.

Para la digestión de las muestras se utilizó una solución de $\mathrm{KOH}$ al 10\%, previamente filtrada $(2.5 \mu \mathrm{m})$, en una proporción de 4:1 (KOH: muestra). Las muestras fueron incubadas a $60{ }^{\circ} \mathrm{C}$ durante $24 \mathrm{~h}$ con agitación continua a $45 \mathrm{rpm}$. Terminada la digestión se procedió a filtrar con una bomba de vacío usando un filtro de porosidad de 6 $\mu \mathrm{m}$ WhatmanTM N. ${ }^{\circ} 3$. Finalmente, el filtro fue secado a $60{ }^{\circ} \mathrm{C}$.

Para la caracterización visual de MP se siguió los criterios de Lusher et al. (2013, 2016). Se tomaron registros numéricos de abundancia de la forma (fibra «plástico delgado o fibroso, y recto», fragmento «partícula de plástico duro e irregular», film «plástico endeble plano y delgado» o pellet «partícula de plástico duro y redondeado») (Sruthy y Ramasamy, 2017) y color (azul, fucsia, verde, negro y blanco). Se utilizó un estereoscopio y microscopio para inspeccionar visualmente cada papel filtro, y se tomó registro fotográfico de lo encontrado.

\section{Análisis Estadístico}

Se determinó el grado de asociación mediante el coeficiente de correlación de Pearson $\left(r_{p}\right)$ y Spearman $\left(r_{s}\right)$ entre la LT y peso del pez vs el número de ítems de MP por especie de pez evaluado. Previamente se verificó la normalidad con las pruebas de Shapiro-Wilk $(\mathrm{n}<50)$ y la prueba de Homogeneidad de Varianzas de Levene; seleccionando asi, la correlación de tipo paramétrica (Pearson, $r_{p}$ ) o no paramétrica (Spearman, $r_{s}$ ) adecuada. Para determinar si hay diferencias entre las especies estudiadas con relación a la cantidad de MP en el tracto digestivo y en las branquias, la LT y el peso, fue empleada la prueba de Kruskall-Wallis $(\mathrm{H})$ con su estadístico Tukey, el cual determinó el nivel de diferencias entre las cinco especies de peces marinos. Fue utilizada la prueba de $\mathrm{t}$ de Student para comparar el número de ítems de MP de tracto digestivo, branquias, LT y peso con relación al sexo por cada especie de pez. Para todo el proceso de datos se empleó el paquete estadístico Statistical Package for the Social Sciences (SPSS) v. 22 para Windows 10. Se calculó para todos los casos un valor de significancia de alfa de 0.05 .

\section{Resultados}

Los datos biométricos de los peces y la cantidad total de MP encontrados en el tracto digesivo y branquias por especie se muestran en el Cuadro 1. Se notó la presencia de MP en el 100\% de los peces. Se observaron diferencias entre las medidas biométricas de los peces, siendo la LT y el peso de $S$. japonicus y $M$. cephalus mayores que las otras tres especies ícticas. Con relación a los MP encontrados en el tracto digestivo, $S$. gigas presentó valores mayores que $I$. conceptionis y $M$. cephalus, y estos a su vez fueron diferentes a $S$. deliciosa y $S$. japonicus. No se observaron diferencias en la cantidad de MP registrados en las branquias entre especies (Cuadro 1).

El Cuadro 2 muestra una correlacion positiva entre la talla de I. conceptionis y MP de tracto digestivo/pez. De igual manera se vio una asociación positiva entre el peso de M. cephalus y los MP del tracto digestivo/pez. Para el resto de las peces no se vio asociación entre la talla y el peso, y los MP del tracto digestivo/pez. Tampoco se vio asociación entre la talla y el peso y MP de las branquias/pez. Asimismo, tampoco hubo diferencia significativa al comparar los peces por sexo dentro de especie entre las partículas de MP por tracto digestivo y branquias, LT y peso (Cuadro 3).

El Cuadro 4 señala que a nivel del tracto digestivo, el MP azul, negro y blanco fueron los tres colores dominantes en las cinco especies de peces marinos. De igual forma, los 
Cuadro 1. Datos ecológicos, biométricos y cantidad de microplástico (MP) en peces marinos de importancia económica en Lima, Perú

\begin{tabular}{|c|c|c|c|c|c|c|c|c|c|}
\hline 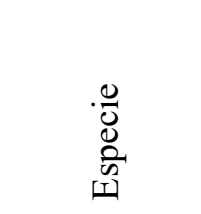 & 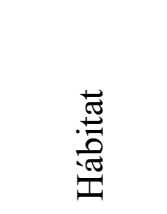 & 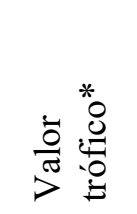 & 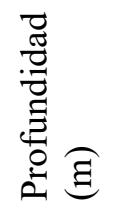 & $\begin{array}{l}\text { की } \\
0 \\
0 \\
0 \\
0\end{array}$ & 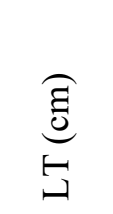 & $\underbrace{E}_{\Sigma}$ & $\vec{E}$ & $\sum^{D}$ & $\sum$ \\
\hline $\begin{array}{l}\text { Sciaena } \\
\text { deliciosa }\end{array}$ & $\begin{array}{l}\text { Bento- } \\
\text { pelágica }\end{array}$ & $3.5 \pm 0.4$ & $0-50$ & $\begin{array}{c}246.2 \pm \\
120.8^{b}\end{array}$ & $\begin{array}{c}27.3^{ \pm} \\
5.3^{\mathrm{b}}\end{array}$ & 294 & 214 & $\begin{array}{l}14.7 \pm \\
10.1^{\mathrm{a}}\end{array}$ & $\begin{array}{c}10.7 \pm \\
6.5^{\mathrm{a}}\end{array}$ \\
\hline $\begin{array}{l}\text { Isacia } \\
\text { conceptionis }\end{array}$ & $\begin{array}{l}\text { Bento- } \\
\text { pelágica }\end{array}$ & $2.9 \pm 0.4$ & $0-50$ & $\begin{array}{c}163.6 \pm \\
26.1^{\mathrm{a}}\end{array}$ & $\begin{array}{c}23.6 \pm \\
1.5^{\mathrm{a}}\end{array}$ & 579 & 196 & $\begin{array}{c}29.0 \pm \\
9.3^{\mathrm{b}}\end{array}$ & $\begin{array}{l}9.8 \pm \\
4.7^{\mathrm{a}}\end{array}$ \\
\hline $\begin{array}{l}\text { Scartichthys } \\
\text { gigas }\end{array}$ & Bentónica & $3.0 \pm 0.4$ & $0-10$ & $\begin{array}{c}156.8 \pm \\
40.6^{\mathrm{a}}\end{array}$ & $\begin{array}{c}22.5 \pm \\
2.2^{\mathrm{a}}\end{array}$ & 878 & 264 & $\begin{array}{l}43.9 \pm \\
14.2^{c}\end{array}$ & $\begin{array}{c}13.2 \pm \\
8.0^{\mathrm{a}}\end{array}$ \\
\hline $\begin{array}{l}\text { Scomber } \\
\text { japonicus }\end{array}$ & Pelágica & $3.4 \pm 0.1$ & $0-100$ & $\begin{array}{c}497.6^{ \pm} \\
62.4^{\mathrm{c}}\end{array}$ & $\begin{array}{c}32.9 \pm \\
3.0^{\mathrm{c}}\end{array}$ & 350 & 171 & $\begin{array}{c}17.5 \pm \\
6.3 \mathrm{a}\end{array}$ & $\begin{array}{l}8.6 \pm \\
6.2^{\mathrm{a}}\end{array}$ \\
\hline $\begin{array}{l}\text { Mugil } \\
\text { cephalus }\end{array}$ & $\begin{array}{l}\text { Bento- } \\
\text { pelágica }\end{array}$ & $2.5 \pm 0.2$ & $0-120$ & $\begin{array}{c}443.9 \pm \\
84.7^{\mathrm{c}}\end{array}$ & $\begin{array}{c}34.4 \pm \\
1.8^{\mathrm{c}}\end{array}$ & 691 & 209 & $\begin{array}{l}34.6 \pm \\
11.8^{b}\end{array}$ & $\begin{array}{c}10.4 \pm \\
3.9^{\mathrm{a}}\end{array}$ \\
\hline $\begin{array}{l}\text { Kruskall- } \\
\text { Wallis (H) }\end{array}$ & & & & 70.71 & 65.82 & & & 61.51 & 5.83 \\
\hline $\mathrm{P}$ & & & & $<0.05$ & $<0.05$ & & & $<0.05$ & $>0.05$ \\
\hline
\end{tabular}

( \pm DE): Desviación estándar, LT: Longitud total del pez, MPTtd: Microplástico total de tracto digesivo, MPTb: Microplástico total de branquias, MPd: Promedio de MP de tracto digestivo, $\mathrm{MPb}$ : Promedio de MP de branquias

$\mathrm{p}=$ significancia. ${ }^{*}=$ valor trofico según lo señalado por Froese y Pauly (2020) en base a lo propuesto por Stergiou y Karpuzi (2002) y Hayden et al. (2019)

Los valores del valor trófico entre $>2.1$ y $<2.9$ indican que es una especie onmívora con preferencias por material vegetal y entre $>2.9$ y $<3.7$ señalan que es una especie onmívora con preferencias por material animal

Cuadro 2. Correlación de Pearson (r) entre cantidad de microplásticos (MP) vs longitud total (LT) y peso en peces marinos de importancia económica en Lima, Perú

\begin{tabular}{|c|c|c|c|c|c|c|c|c|}
\hline \multirow{3}{*}{ Especie } & \multicolumn{4}{|c|}{ Tracto digestivo } & \multicolumn{4}{|c|}{ Branquias } \\
\hline & \multicolumn{2}{|c|}{ LT } & \multicolumn{2}{|c|}{ Peso } & \multicolumn{2}{|c|}{$\mathrm{LT}$} & \multicolumn{2}{|c|}{ Peso } \\
\hline & $\mathrm{r}$ & $\mathrm{p}$ & $\mathrm{r}$ & $\mathrm{p}$ & $\mathrm{r}$ & $\mathrm{p}$ & $\mathrm{r}$ & $P$ \\
\hline Sciaena deliciosa & -0.28 & 0.23 & -0.27 & 0.24 & -0.19 & 0.41 & -0.21 & 0.38 \\
\hline Isacia conceptionis & 0.49 & 0.03 & 0.29 & 0.22 & 0.02 & 0.92 & 0.12 & 0.60 \\
\hline Scartichthys gigas & 0.34 & 0.14 & 0.20 & 0.40 & 0.25 & 0.29 & 0.09 & 0.67 \\
\hline Scomber japonicus & -0.08 & 0.72 & -0.24 & 0.32 & 0.07 & 0.76 & -0.23 & 0.33 \\
\hline Mugil cephalus & 0.30 & 0.19 & 0.49 & 0.03 & -0.17 & 0.47 & -0.11 & 0.63 \\
\hline
\end{tabular}

$p=$ significancia. Valores en negrita señalan asociación entre las variables $(p<0.05)$ 
Cuadro 3 Prueba de $t$ Student para el sexo respecto a los tipos de microplásticos (MP) en peces marinos de importancia económica en Lima, Perú

\begin{tabular}{lcccccccc}
\hline \multirow{2}{*}{ Especie } & \multicolumn{2}{c}{$\begin{array}{c}\text { Tracto } \\
\text { digestivo }\end{array}$} & \multicolumn{2}{c}{ Branquias } & \multicolumn{2}{c}{$\begin{array}{c}\text { Longitud total } \\
\text { (LT) }\end{array}$} & \multicolumn{2}{c}{ Peso } \\
\cline { 2 - 10 } & $\mathrm{t}$ & $p$ & $\mathrm{t}$ & $p$ & $\mathrm{t}$ & $p$ & $\mathrm{t}$ & $p$ \\
\hline Sciaena deliciosa & 0.55 & 0.59 & 0.31 & 0.76 & 0.04 & 0.96 & 0.04 & 0.96 \\
Isacia conceptionis & 0.55 & 0.59 & 0.24 & 0.81 & 1.16 & 0.12 & 0.86 & 0.40 \\
Scartichthys gigas & 0.67 & 0.51 & 0.43 & 0.66 & 0.52 & 0.61 & 0.72 & 0.48 \\
Scomber japonicus & 0.86 & 0.40 & 1.32 & 0.20 & 0.12 & 0.90 & 0.62 & 0.54 \\
Mugil cephalus & 0.63 & 0.68 & 1.55 & 0.14 & 0.84 & 0.42 & 0.56 & 0.57 \\
\hline
\end{tabular}

$p=$ significancia

MP azul, negro y fucsia en las branquias fueron los colores más abundantes en las cinco especies ícticas. En el Cuadro 5 se observa una mayor abundancia de fibra, seguida de fragmento, film y pellet por especie de pez a nivel del tracto digestivo. En el caso de las branquias el único tipo de MP presente fue la fibra. Fotografías representativas de los colores de MP por forma fibra, fragmento y film son mostrados en las figuras 1, 2 y 3 .

\section{Discusión}

Todos los peces, independientemente de sus hábitos de alimentación, presentaron MP en el tracto digestivo y en las branquias. De las cinco especies ícticas, $S$. gigas fue la que presentó más partículas de MP en el tracto digestivo, seguido por $M$. cephalus, I. conceptionis, S. japonicus y S. deliciosa. Para explicar las cantidades de MP halladas en los tractos digestivos debe considerarse como factores importantes parámetros bioecológicos como el hábitat, el rol trófico en los peces evaluados y la profundidad en el medio marino (Cheung et al., 2018; De-la-Torre et al., 2019a; Bucol et al.,
2020; Pereira et al., 2020; Weis, 2020). El valor trófico según Froese y Pauly (2020) con base a lo señalado por Stergiou y Karpuzi (2002) y Hayden et al. (2019) no mostró un patrón claro con relación a la cantidad de MP observada. Así, S. gigas e I. conceptionis consideradas onmívoras con preferencias por material animal y $M$. cephalus como una especie onmívora con preferencias por material vegetal fueron las que presentaron mayores cantidades de MP/pez. En el caso de $S$. gigas, el hábitat bentónico rocoso y una preferencia a ambientes de menor profundidad $(0-10 \mathrm{~m})$ y cercanos al ambiente urbano de la ciudad de Lima parecen explicar la presencia de una mayor cantidad de MP/pez.

La mayoría de las especies de peces de este estudio tienen un habitat arenoso-rocoso poco profundo y cercanos a las playas al ser bentónicas y bentopelágicas, a excepción de $S$. japonicus que es pelágica (Froese y Pauly, 2020). Se podría deducir entonces que la cantidad de partículas de MP presentes en el tracto digestivo y branquias pudiera estar relacionada con la proximidad a las zonas urbanas del ecosistema marino (Peters y Bratton, 2016; Murphy et al., 2017; Halstead et al., 2018). 
La variación en las tasas de ingestión parece estar altamente relacionada con la disponibilidad de MP en el medio ambiente, que se ve afectada por factores como la profundidad, distancia a la costa o urbanización del área (De-la-Torre et al., 2019ab; Bermúdez-Guzmán et al., 2020). Bråte et al. (2016) concluyen que los peces que tienen hábitos lejanos a las zonas rocosas o playas presentan menor cantidad de MP en comparación con los que se encuentran cercanos al ambiente rocoso. Esto podría explicar que la especie pelágica $S$. japonicus haya presentado la menor cantidad de MP/pez. De otra parte, Lusher et al. $(2013,2016)$ evidencian que el número de MP por individuo va de $0 \mathrm{a}$ 21 en los peces marinos examinados, resultados menores a los del presente estudio donde el número de MP encontradas en el tracto digestivo varia entre 14.70 a $43.90 \mathrm{MP} /$ pez.

La relación de peso y LT de los peces con la cantidad de MP en el tracto digestivo no resultó ser significativa para la mayoría de las especies. Solo se observó una correlacion positiva entre la LT de $I$. conceptionis y los MP del tracto digestivo/ pez. De igual manera, se vio una asociación positiva entre el peso de $M$. cephalus y MP del tracto digestivo/pez. Estos resultados concuerdan con otros estudios (Phillips y Bonner, 2015; Calderón y Hansen, 2018), en los cuales no se encontró una correlación significativa entre estas variables con la presencia de MP. Pazos et al. (2017), de otra parte, obtuvieron asociación entre estas dos variables con los MP en tracto digestivo.

La relación entre LT y peso de los peces con la cantidad de MP en branquias no fue significativa. Algunos autores señalan que una mayor cantidad de MP en branquias en peces de menor tamaño podría explicarse porque estos realizan una mayor tasa de respiración (Au et al., 2017) y, en consecuencia, una mayor filtración de agua potencialmente contaminada con MP, lo que aumentaría la probabilidad de acumulación de MP (Weis, 2020). Sin embargo, las especies evaluadas en el presente estudio no mostraron una relación entre las medidas biométricas con el número de partículas de MP/branquia. Tampoco se observó diferencias entre hembras y machos, resultado similar al obtenido por Parton et al. (2020).

McNeish et al. (2018) mencionan que la estrategia de alimentación de los peces está relacionada con la transferencia trófica de las partículas de plástico, por lo que estas especies podrían presentar un riesgo para otros organismos de diferentes niveles tróficos, peces de mayor tamaño, aves y mamíferos que se alimentan de otros peces, como $S$. deliciosa que se alimenta de la anchoveta (Parton et al., 2020; Weis, 2020). Calderón y Hansen (2018) mencionan que probablemente los peces omnívoros con preferencia a alimentos animales tengan una mayor abundancia de partículas MP, lo que coincidiría con este estudio, ya que $S$. gigas presentó 43.9/pez de MP.

En cuanto a los colores de MP, el más abundante fue el azul, seguido de negro y blanco, fucsia y verde. Algunos autores mencionan que el color de MP estaría relacionado con su composición química o absorción de tóxicos en el mar (Parton et al., 2020; Weis, 2020). Worm et al. (2017) han demostrado que los MP liberan sustancias tóxicas, que incluyen monómeros residuales, plastificantes y agentes colorantes, entre otros aditivos, que pueden ingerirse y producir bioacumulación. Dado que los peces tienen un método de alimentación selectivo, Rummel et al. (2016) y Lusher et al. (2016) sugieren que los MP se pueden asimilar a través de la ingesta de presas contaminadas o al ingerirlos accidentalmente cuando se confunden con alimento (Bucol et al., 2020; Weis, 2020).

El tipo de MP más abundante en las cinco especies, tanto en tracto digestivo como en las branquias, fueron las fibras, lo que se registra en la mayoría de los estudios (Bellas et al., 2016; Neves et al., 2015; Phuong et al., 2016; Pazos et al., 2017; Chan et al., 2019; Parton et al., 2020). Song et al. (2015) 
Cuadro 4. Cantidad de partículas de microplásticos (MP) por colores en peces marinos de importancia económica en Lima, Perú

\begin{tabular}{|c|c|c|c|c|c|c|c|c|c|c|c|c|}
\hline \multirow[b]{2}{*}{ Especie de pez } & \multirow[b]{2}{*}{ 胥 } & \multicolumn{5}{|c|}{ Tracto digestivo } & \multirow[b]{2}{*}{$\stackrel{\vec{Q}}{\vec{E}}$} & \multicolumn{5}{|c|}{ Branquias } \\
\hline & & $\overline{\bar{Z}}$ & 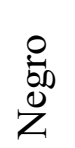 & 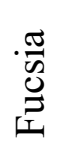 & $\frac{⿱ 乛}{0}$ & $\frac{0}{\stackrel{\Xi}{\Xi}}$ & & $\overline{\mathrm{z}}$ & $\begin{array}{l}\text { O } \\
\stackrel{0}{0} \\
Z\end{array}$ & 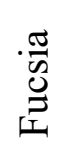 & $\frac{0}{\frac{0}{0}}$ & $\begin{array}{l}\text { O্ } \\
\frac{\vec{E}}{m}\end{array}$ \\
\hline Sciaena deliciosa & 294 & 26 & 39 & 8 & 6 & 21 & 214 & 49 & 25 & 18 & 8 & 0 \\
\hline Isacia conceptionis & 579 & 55 & 16 & 10 & 4 & 15 & 196 & 36 & 32 & 12 & 3 & 17 \\
\hline Scartichthys gigas & 878 & 65 & 11 & 10 & 0 & 13 & 264 & 52 & 20 & 17 & 3 & 8 \\
\hline Scomber japonicus & 350 & 58 & 20 & 7 & 3 & 13 & 171 & 29 & 15 & 17 & 0 & 40 \\
\hline Mugil cephalus & 691 & 46 & 13 & 21 & 3 & 18 & 209 & 47 & 17 & 31 & 5 & 0 \\
\hline Total de MP & & 250 & 99 & 48 & 16 & 80 & & 213 & 106 & 95 & 19 & 65 \\
\hline
\end{tabular}

MPTtd: Microplástico total de tracto digesivo, MPTb: Microplástico total de branquias

Cuadro 5. Cantidad de partículas de microplásticos (MP) por forma en peces marinos de importancia económica en Lima, Perú

\begin{tabular}{|c|c|c|c|c|c|c|c|c|c|c|}
\hline \multirow[b]{2}{*}{ Especie } & \multirow[b]{2}{*}{$\begin{array}{l}\stackrel{D}{E} \\
E \\
\sum\end{array}$} & \multicolumn{4}{|c|}{ Tracto digestivo } & \multirow[b]{2}{*}{$\stackrel{\vec{Q}}{E}$} & \multicolumn{4}{|c|}{ Branquias } \\
\hline & & $\stackrel{\pi}{0}$ & 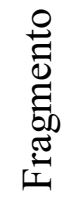 & $\underset{\Xi}{\Xi}$ & $\frac{\vec{U}}{\overline{0}}$ & & 营 & 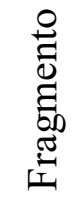 & $\Xi$ & $\frac{\overrightarrow{0}}{\overline{0}}$ \\
\hline Sciaena deliciosa & 294 & 266 & 4 & 24 & 0 & 214 & 214 & 0 & 0 & 0 \\
\hline Isacia conceptionis & 579 & 541 & 23 & 15 & 0 & 196 & 196 & 0 & 0 & 0 \\
\hline Scartichthys gigas & 878 & 845 & 22 & 8 & 3 & 264 & 264 & 0 & 0 & 0 \\
\hline Scomber japonicus & 350 & 315 & 5 & 30 & 0 & 171 & 171 & 0 & 0 & 0 \\
\hline Mugil cephalus & 691 & 626 & 25 & 38 & 2 & 209 & 209 & 0 & 0 & 0 \\
\hline Total de MP & 2792 & 2593 & 79 & 115 & 5 & 1054 & 1054 & 0 & 0 & 0 \\
\hline
\end{tabular}

MPTtd: Microplástico total de tracto digesivo, MPTb: Microplástico total de branquias

sugieren identificar debidamente los MP ya que algunas fibras parecen plásticas, pero en realidad son de algodón.

Las piezas de artes de pesca son una de las fuentes de MP. Los principales artes de pesca que se emplean en Perú para la captura son el boliche (malla de $38 \mathrm{~mm}$ ) y la cortina (malla de $65 \mathrm{~mm}$ ) (IMARPE, 2000) que, por acción de fuerza mecánica, rayos solares, fricción, etc., se podrían fragmentar $\mathrm{y}$, así de manera directa, llegar al mar mezclándose con el alimento de los peces (Auta et al., 2017; Pozo et al., 2019; Parton et al., 2020; Wang et al., 2020a). 


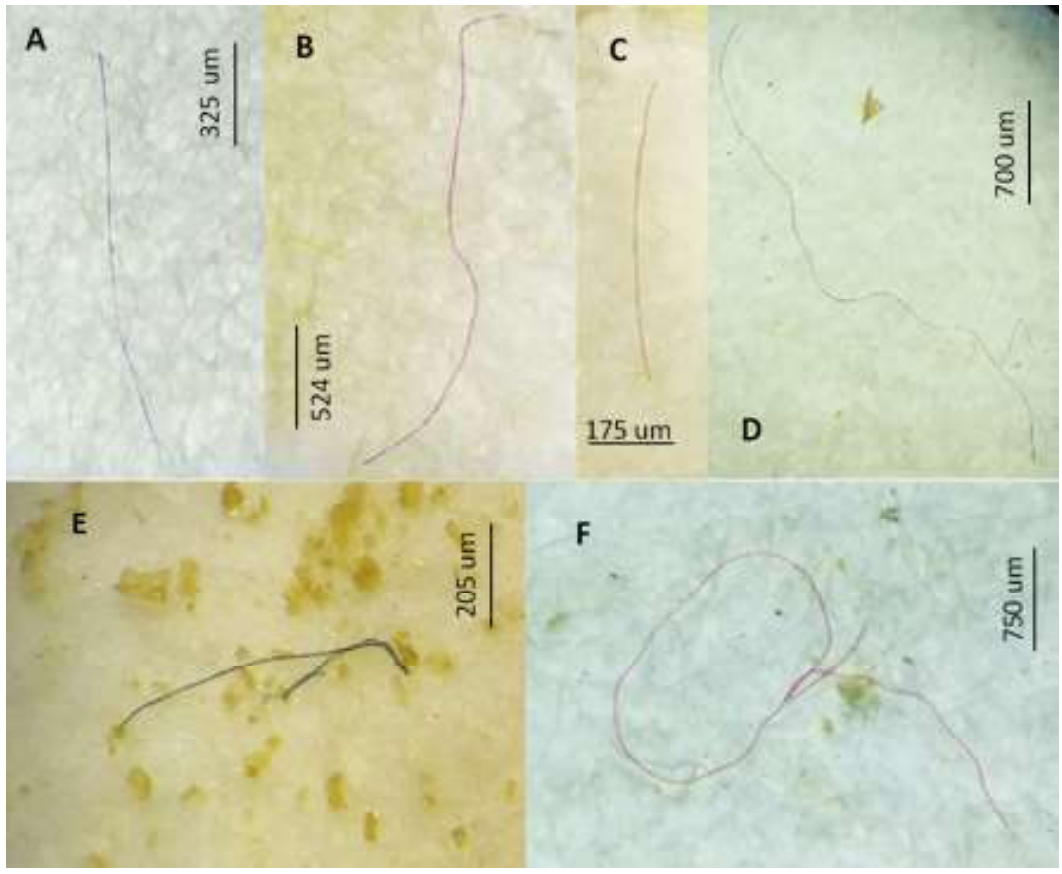

Figura 1. Microplástico de tipo fibra según el color. A: azul, B y F: fucsia, C: Verde, D: blanco, E: negro

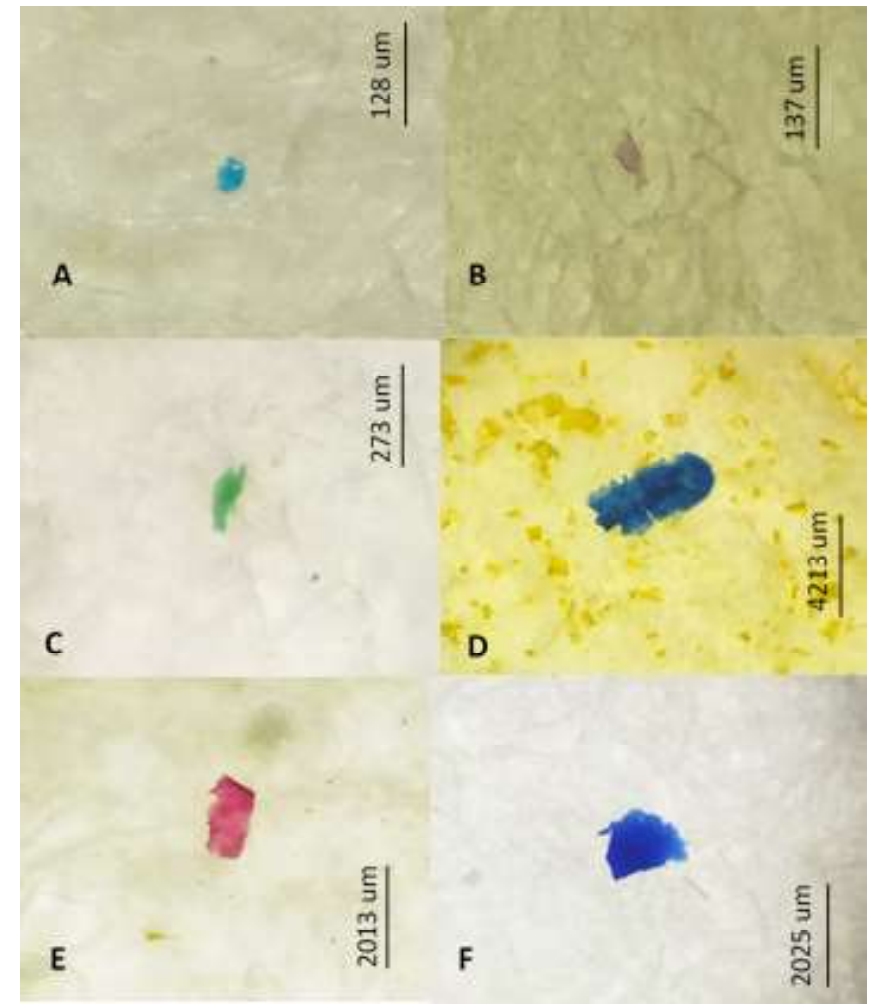

Figura 2. Microplástico de tipo fragmento según el color. A, D y F: azul; B y E: fucsia; C: verde 


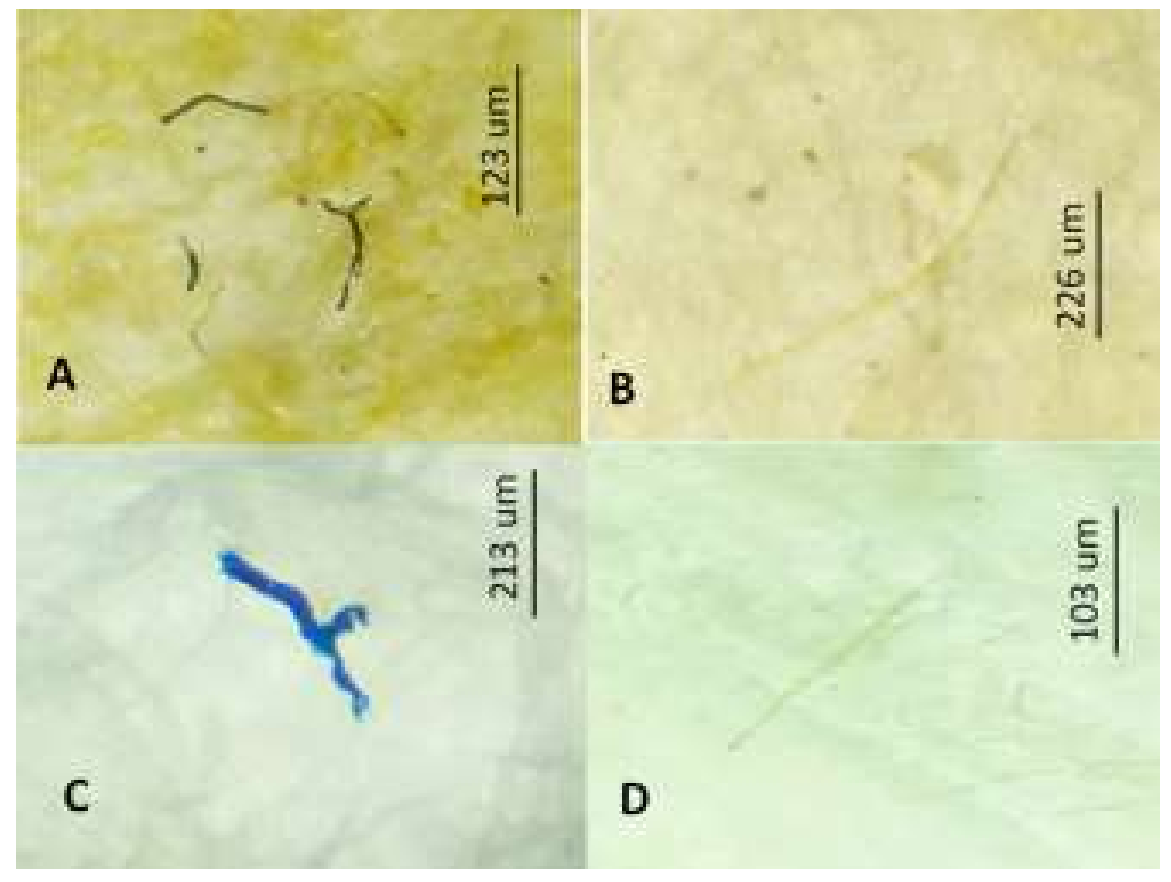

Figura 3. Microplásticos de tipo film según el color. A: Negro, B: verde, C: Azul, D: blanco

Galloway y Lewis (2016) comprobaron que las células y tejidos humanos absorben y filtran los contaminantes químicos relacionados a la ingesta de pescados y mariscos con MP, pero debido a la falta de datos y conocimientos científicos es difícil evaluar el nivel de riesgo para la salud humana. Correia-Prata (2018) ha demostrado que las particulas de MP en el aire generan disnea (dificultad para respirar por inflamación de fosas nasales), algunas personas pueden ser mas sensibles de otras, pudiéndose desarrollar enfermedades respiratorias, anque consideran que la concentración de MP en el ambiente aun son bajos.

\section{Conclusiones}

- Se observó la presencia de microplásticos (MP) en todos los peces de las cinco especies estudiadas de importancia eco- nómica en Lima, Perú (S. deliciosa, I. conceptionis, S. gigas, M. cephalus y S. japonicus).

- Los MP se encuentran tanto en el tracto digestivo por el consumo de alimento, como en las branquias por filtración del agua.

- Los MP encontrados en el tracto digestivo de $S$. gigas presentaron valores mayores que $I$. conceptionis y $M$. cephalus.

- No se observaron diferencias en la cantidad de MP registrados en las branquias para las cinco especies de peces.

- Se observó una correlacion positiva entre la longitud de I. conceptionis y MP de tracto digestivo/pez, y entre el peso de $M$. cephalus y MP del tracto digestivo/pez.

- El azul, negro y blanco fueron los tres colores dominantes de los MP en el tracto digestivo y el azul, negro y fucsia fueron los más abundantes en las branquias. 
- A nivel del tracto digestivo, según el tipo de MP, se encontró una mayor proporción fibra, seguido de fragmentos y film, mientras que en branquias solo se encontraron fibras

\section{Literatura Citada}

1. Anderson JC, Park BJ, Palace VC. 2016. Microplastics in aquatic environments: Implications for Canadian ecosystems. Environ Pollut 218: 269-280. doi: 10.1016/j.envpol.2016.06.074

2. Au SY, Lee CM, Weinstein JE, van den Hurk, P, Klaine SJ. 2017. Trophic transfer of microplastics in aquatic ecosystems: identifying critical research needs. Integr Environ Asses 13: 505-509. doi: 10.1002/ieam.1907

3. Auta HS, Emenike CU, Fauziah SH. 2017. Distribution and importance of microplastics in the marine environment: a review of the sources, fate, effects, and potential solutions. Environ Int 102: 165-176. doi: 10.1016/j.envint.2017.02.013

4. Barboza LGA, Lopes C, Oliveira $P$, Bessa F, Otero $V$, Henriques $B$, Raimundo J, et al. 2019. Microplastic in wild fish from North East Atlantic Ocean and its potential for causing neurotoxic effects, lipid oxidative damage, and human health risks associated with ingestion exposure. Sci Total Environ 717: 134625. doi: 10.1016/ j.scitotenv.2019.134625

5. Barboza LGA, Vethaak AD, Lavorante BRBO, Lundebye AK, Guilhermino L, Reaimundo J, et al. 2018. Marine microplastic debris: an emerging issue for food security, food safety and human health. Mar Pollut Bull 133: 336-348. doi: 10.1016/j.marpolbul.2018.05.047

6. Battaglia P, Pedà C, Musolino S, Esposito V, Andaloro $F$, Romeo $T$. 2016. Diet and first documented data on plastic ingestion of Trachinotus ovatus L. 1758 (Pisces: Carangidae) from the Strait of Messina (central Mediterranean Sea). Ital J Zool 83: 121129. doi: $10.1080 / 11250003.2015 .-1114157$

7. Bellas J, Martínez-Armental J, Martínez-Cámara A, Besada V, Martínez-Gómez C. 2016. Ingestion of microplastics by demersal fish from the Spanish Atlantic and Mediterranean coasts. Mar Pollut Bull 109: 55-60. doi: 10.1016/j.marpolbul.2016.06.026

8. Bergmann M, Wirzberger V, Krumpen T, Lorenz C, Primpke S, Tekman MB, Gerdts G. 2017. High quantities of microplastic in Arctic deep-sea sediments from the Hausgarten observatory. Environ SciTechnol 51: 11000-11010. doi: 10.1021/ acs.est.7b0-3331

9. Bermúdez-Guzmán L, AlpízarVillalobos C, Gatgens-García J, Jiménez-Huezo G, Rodríguez-Arias M, Molina H, Villalobos J, et al. 2020. Microplastic ingestion by a herring Opisthonema sp, in the Pacific coast of Costa Rica. Reg Stud Mar Sci 38: 101367. doi: 10.1016/j.rsma.2020.101367

10. Bråte ILN, Eidsvoll DP, Steindal CC, Thomas KV. 2016. Plastic ingestion by Atlantic cod (Gadus morhua) from the Norwegian coast. Mar Pollut Bull 112: 105-110. doi: 10.1016/j.marpolbul.2016.08.034

11. Bucol LA, Romano EF, Cabcaban SM, Siplona LMD, Madrid GC, Bucol, AA, Polidoro B. 2020. Microplastics in marine sediments and rabbitfish (Siganus fuscescens) from selected coastal areas of Negros Oriental, Philippines. Mar Pollut Bull 150: 110685. doi: 10.1016/j.marpolbul.2019.110685

12. Calderón E, Hansen P. 2018. Microplastics in the digestive tract of fish from the estuary Ciénaga Grande de Santa Marta, Colombia. Master thesis. Denmark: Roskilde University. $32 \mathrm{p}$.

13. Chan HSH, Dingle C, Not C. 2019. Evidence for non-selective ingestion of microplastic in demersal fish. Mar Pollut Bull 149: 110523. doi: 10.1016/j.marpolbul.2019.110523 
14. Cheung L, Lui C, Fok L. 2018. Microplastic contamination of wild and captive flathead Grey mullet (Mugil cephalus). Int J Environ Res Pu 15: 597. doi: 10.3390/ijerph15040597

15. Cincinelli A, Scopetani C, Chelazzi D, Lombardini E, Martellini T, Katsoyiannis A, Fossi MC, Corsolini S. 2017. Microplastic in the surface waters of the Ross Sea (Antarctica): occurrence, distribution and characterization by FTIR. Chemosphere 175: 391400. doi: $10.1016 /$ j.chemosphere.2017.02.024

16. Codina-García M, Militão T, Moreno J, González-Solís J. 2013. Plastic debris in Mediterranean seabirds. Mar Pollut Bull 77: 220-226. doi: 10.1016/ j.marpolbul.2013.10.002

17. Cole M, Lindeque P, Halsband C, Galloway TS. 2011. Microplastics as contaminants in the marine environment: a review. Mar Pollut Bull 62: 2588-2597. doi: 10.1016/j.marpolbul.2011.09.025

18. Correia-Prata J. 2018. Airborne microplastics: consequences to human health? Environ Pollut 234: 115-126. doi: 10.1016/j.envpol.2017.11.043

19. Cózar A, Echevarria F, GonzalezGordillo JI, Irigoien X, Ubeda B, Hernandez-Leon S, Palma AT, et al. 2014. Plastic debris in the open ocean. P Natl Acad Sci USA 111: 10239-10244. doi:10.1073/pnas.1314705111

20. Cózar A, Martí E, Duarte CM, Garcíade-Lomas J, van Sebille E, Ballatore TJ, Eguiluz VM, et al. 2017. The Arctic Ocean as a dead end for floating plastics in the North Atlantic branch of the Thermohaline Circulation. Sci Adv 3: e1600582. doi: 10.1126/sciadv.1600582

21. Davison P, Asch RG. 2011. Plastic ingestion by mesopelagic fishes in the North Pacific Subtropical Gyre. Mar Ecol Prog Ser 432: 173-180. doi: 10.3354/ meps09142

22. De-la-Torre GE, Dioses-Salinas DC, Pérez-Baca BL, Santillán L. 2019a. Microplastic abundance in three com- mercial fish from the coast of Lima, Peru. Braz J Nat Sci 2: 171-171. doi: 10.31415/bjns.v2i3.67

23. De-la-Torre G, Mendoza-Castilla L, Pilar R. 2019b. Microplastic contamina-tion in market bivalve Argopecten purpuratus from Lima, Peru. Manglar 16: 85-89. doi:10.17268/manglar.2019.012

24. De-la-Torre G, Dioses-Salinas D, Castro J, Antay R, Fernández $N$, Espinoza-Morriberón D, Saldaña-Serrano $M .2020$. Abundance and distribution of microplastics on sandy beaches of Lima, Peru. Mar Pollut Bull 151: 110877. doi: 10.1016/j.marpolbul.2019.110877

25. Frias JPGL, Nash R. 2018. Microplastics: Finding a consensus on the definition. Mar Pollut Bull 138: 145147. doi: 10.1016/j.marpolbul.2018.11.022

26. Froese R, Pauly D. 2020. FishBase. World Wide Web electronic publication. www.fishbase.org

27. Gallo F, Fossi C, Weber R, Santillo D, Sousa J, Ingram I, Nadal A, Romano D. 2018. Marine litter plastics and microplastics and their toxic chemicals components: the need for urgent preventive measures. Environ Sci Eur 30: 1314. doi: 10.1186/s12302-018-0139-z

28. Galloway T, Lewis C. 2016. Marine microplastics spell big problems for future generations. P Natl Acad Sci USA 113: 2331-2333. doi: 10.1073/pnas.1600715113

29. [GESAMP] Group of Experts on the Scientific Aspects of Marine Environmental Protection. 2016. Sources, fate and effects of microplastics in the marine environment: part two of a global assessment. In: Kershaw PJ, Rochman CM (eds). IMO/FAO/ UNESCOIOC/UNIDO/WMO/IAEA/ UN/UNEP/UNDP Joint Group of Experts on the Scientific Aspects of Marine Environmental Protection. 220 p. 
30. Halstead JE, Smith JA, Carter EA, Lay PA, Johnston EL. 2018. Assessment tools for microplastics and natural fibres ingested by fish in an urbanised estuary. Environ Pollut 234: 552-561. doi: 10.1016/j.envpol.2017.-11.085.

31. Hayden B, Palomares MLD, Smith BE, Poelen JH. 2019. Biological and environmental drivers of trophic ecology in marine fishes - a global perspective. Sci Rep 9: 11415. doi:10.1038/s41598019-47618-2

32. Iannacone J, Huyhua A, Alvariño L, Valencia F, Principe F, Minaya D, Ortega J, et al. 2019. Microplásticos en la zona de marea alta y supralitoral de una playa arenosa del litoral costero del Perú. Biologist 17: 335-346. doi: 10.24039/rtb2019172369

33. [IMARPE] Instituto del Mar del Perú. 2000. Aspectos biológico-pesqueros de la Lorna (Sciaena deliciosa) y el Machete (Ethimidium maculatum) en el área de Huacho durante un periodo frio y otro cálido. Callao, Perú: Informe N. ${ }^{\circ}$ $128.11 \mathrm{p}$.

34. Lönnstedt OO, Eklöv P. 2016. Environmentally relevant concentrations of microplastic particles influence larval fish ecology. Science 352: 1213-1216. doi: $10.1126 /$ science.aad 8828

35. Lusher A, McHugh M, Thompson R. 2013. Ocurrence of microplastics in the gastrointestinal tract of pelagic and demersal fish from the English Channel. Mar Pollut Bull 67: 94-99. doi: 10.1016/ j.marpolbul.2012.11.028

36. Lusher AL, O'Donnell C, Officer R, O'Connor I. 2016. Microplastic interactions with North Atlantic mesopelagic fish. ICES J Mar Sci 73: 1214-1225. doi: 10.1093/icesjms/fsv241

37. Lusher AL, Hernandez-Milian G. 2018. Microplastic extraction from marine vertebrate digestive tracts, regurgitates and scats: A protocol for researchers from all experience levels. Bio-protocol 8: 1-12. doi: 10.21769/ BioProtoc.3086
38. Maes T, Meulen MDVD, Devriese LI, Leslie HA, Huvet A, Frère L, Robbens J, et al. 2017. Microplastics baseline surveys at the water surface and in sediments of the north-East Atlantic. Front Mar Sci 4: 135. doi: 10.3389/ fmars.2017.00135

39. McGoran AR, Clark PF, Morritt D. 2017. Presence of microplastic in the digestive tracts of European flounder, Platichthys flesus, and European smelt, Osmerus eperlanus, from the River Thames. Environ pollut 220: 744-751. doi: 10.1016/j.envpol.2016.09.078

40. McNeish RE, Kim LH, Barrett HA, Mason SA, Kelly JJ, Hoellein TJ. 2018. Microplastic in riverine fish is connected to species traits. Sci Rep 8: 11639. doi:10.1038/s41598-018-29980-9

41. Mu J, Qu L, Jin F, Zhang S, Fang $C$, Ma X, Zhang W, Huo C, Cong $Y$, Wang J. 2019. Abundance and distribution of microplastics in the surface sediments from the northern Bering and Chukchi seas. Environ Pollut 245: 122-130. doi: 10.1016/j.envpol.2018.10.097

42. Murphy F, Russell M, Ewins C, Quinn B. 2017. The uptake of macroplastic \& microplastic by demersal \& pelagic fish in the Northeast Atlantic around Scotland. Mar Pollut Bull 122: 353-359. doi: 10.1016/j.marpolbul.2017.06.073

43. Neves D, Sobral P, Ferreira JL, Pereira T. 2015. Ingestion of microplastics by commercial fish off the Portuguese coast. Mar Pollut Bull 101: 119-126. doi: 10.1016/j.marpolbul.2015.11.008

44. Ory N, Chagnon C, Felix F, Fernández C, Ferreira JL, Gallardo C, Garcés-Ordóñez O, et al. 2018. Low prevalence of microplastic contamination in planktivorous fish species from the southeast Pacific Ocean. Mar Pollut Bull 127: 211-216. doi: 10.1016/ j.marpolbul.2017.12.016 
45. Parton KJ, Godley BJ, Santillo D, Tausif M, Omeyer LCM, Galloway TS. 2020. Investigating the presence of microplastics in demersal sharks of the North East Atlantic. Sci Rep 10: 12204. doi:.1038/s41598-020-68680-1

46. Pazos RS, Maiztegui T, Colautti DC, Paracampo AH, Gómez N. 2017. Microplastics in gut contents of coastal freshwater fish from Río de la Plata estuary. Mar Pollut Bull 122: 85-90. doi: 10.1016/j.marpolbul.2017.06.007

47. Pereira JM, Rodríguez Y, BlascoMonleon S, Porter A, Lewis C, Pham CK. 2020. Microplastic in the stomachs of open-ocean and deep-sea fishes of the North-East Atlantic. Environ Pollut 265: 115060. doi: 10.1016/j.envpol.2020.115060

48. Peters CA, Bratton SP. 2016. Urbanization is a major influence on microplastic ingestion by sunfish in the Brazos River Basin, Central Texas, USA. Environ Pollut 210: 380-387. doi: 10.1016/ j.envpol.2016.01.018.

49. Phillips MB, Bonner TH. 2015. Occurrence and amount of microplastic ingested by fishes in watersheds of the Gulf of Mexico. Mar Pollut Bull 100: 264269. doi: 10.1016/j.marpolbul.2015.08.041

50. Phuong NN, Zalouk-Vergnoux A, Poirier L, Kamari A, Châtel A, Mouneyrac C, Lagarde F. 2016. Is there any consistency between the microplastics found in the field and those used in laboratory experiments? Environ Pollut 211: 111-123. doi: 10.1016/ j.envpol.2015.12.035

51. Pozo K, Gomez V, Torres M, Vera L, Nuñez D, Oyarzún P, Mendoza G, Clarke, B, Fossi, M, Baini M, Poibylováa P, Klánováa J. 2019. Presence and characterization of microplastics in fish of commercial importance from the Biobío region in central Chile. Mar Pollut Bull 140: 315-319. doi: 10.1016/j.marpolbul.2019.-01.025
52. Purca S, Henostroza A. 2017. Presencia de microplásticos en cuatro playas arenosas de Perú. Rev Peru Biol 24: 101106. doi: 10.15381/rpb.v24i1.12724.

53. Romeo T, Pietro B, Peda C, Consoli P, Andaloro F, Fossi MC. 2015. First evidence of presence of plastic debris in stomach of large pelagic fish in the Mediterranean Sea. Mar Pollut Bull 95: 358-361. doi: 10.1016/j.marpolbul.2015.04.048

54. Royer S-J, Ferro'n S, Wilson ST, Karl DM. 2018. Production of methane and ethylene from plastic in the environment. Plos One 13: e0200574. doi: 10.1371/ journal.pone.0200574

55. Rummel CD, Löder M, Fricke NF, Lang T, Griebeler EM, Janke M, Gerdts G. 2016. Plastic ingestion by pelagic and demersal fish from the North Sea and Baltic Sea. Mar Pollut Bull 102: 134-141. doi: 10.1016/j.marpolbul.2015.11.043

56. Savoca S, Capillo G, Mancuso M, Bottari T, Crupi R, Branca C, Romano $V$, et al. 2019. Microplastics occurrence in the Tyrrhenian waters and in the gastrointestinal tract of two congener species of seabreams. Environ Toxicol Phar 67: 35-41. doi: 10.1016/ j.etap.2019.01.011

57. Song YK, Hong SH, Jang M, Han GM, Rani M, Lee J, Shim WJ. 2015. A comparison of microscopic and spectroscopic identification methods for analysis of microplastics in environmental samples. Mar Pollut Bull 93: 202-209. doi: 10.1016/j.marpolbul.2015.01.015

58. Sruthy S, Ramasamy EV. 2017. Microplastic pollution in Vembanad Lake, Kerala, India: the first report of microplastics in lake and estuarine sediments in India. Environ Pollut 222: 315-322. doi: 10.1016/j.envpol.2016.12.038

59. Stergiou KI, Karpouzi VS. 2002. Feeding habits and trophic levels of Mediterranean fish. Rev Fish Biol Fisher 11: 217-254. doi:10.1023/A:10205567-22822 
60. Strungaru SA, Jijie R, Nicoara M, Plavan G, Faggio C. 2018. Micro (nano) plastics in freshwater ecosystems: abundance, toxicological impact and quantification methodology. TRACTrend Anal Chem 110: 116-128. doi: 10.1016/j.trac.2018.10.025

61. Suaria G, Avio CG, Mineo A, Lattin GL, Magaldi MG, Belmonte G, Moore CJ, Regoli F, Aliani S. 2016. The Mediterranean plastic soup: synthetic polymers in Mediterranean surface waters. Sci Rep 6: 37551. doi:10.1038/ srep37551

62. Valencia-Velasco F, GuablocheZuñiga A, Alvariño L, Iannacone J. 2020. Estandarización de un protocolo para evaluar microplásticos en bivalvos marinos en el departamento de Lima, Perú. Biologist 18: 119-134. doi: 10.24039/ rtb2020181478

63. Wang W, Gao H, Jin S, Li R, Na G 2019. The ecotoxicological effects of microplastics on aquatic food web, from primary producer to human: A review. Ecotox Environ Safe 173: 110-117. doi: 10.1016/j.ecoenv.2019.01.113

64. Wang YL, Lee YH, Chiu IJ, Lin YF, Chiu HW. 2020a. Potential impact of plastiuc nanomaterials and micromaterials on the food chain and human health. Int J Mol Sci 21: 1727. doi: 10.3390/ijms21051727

65. Wang W, Ge J, Yu, X. $2020 b$. Bioavailability and toxicity of microplastics to fish species: a review. Ecotox Environ Safe 189:109913. doi: 10.1016/ j.ecoenv.2019.109913

66. Weis JS. 2020. Aquatic microplastic research - Acritique and suggestions for the future. Water 12: 1475. doi: 10.3390/ w12051475

67. Worm B, Lotze HK, Jubinville I, Wilcox C, Jambeck J. 2017. Plastic as a persistent marine pollutant. Annu Rev Env Resour 42: 1-26. doi: 10.1146/ annurev-environ-102016-060700 